\title{
Los ß-bloqueantes no son un tratamiento de primera línea en la hipertensión del anciano
}

Are ß-blockers efficacious as first-line therapy for hypertension in the elderly? A systematic Review.

Messerli FH, Grossman E, Goldbourt. JAMA 1998;279:1903-1907.

\section{Objetivo}

Valorar en hipertensos ancianos la eficacia antihipertensiva de los ß- bloqueantes y sus efectos sobre la morbimortalidad, comparada con diuréticos.

\section{Fuente de datos y selección de estudios}

Búsqueda por MEDLINE y CARDLINE entre 1966 y 1998 de ensayos randomizados controlados con placebo, de al menos un año de duración, que usaran ß-bloqueantes o diuréticos como tratamiento de primera línea en hipertensos ancianos y que reportaran resultados de morbilidad y mortalidad.

\section{Resultados principales}

Se seleccionaron 10 de 12 ensayos (se excluyeron 2 de pacientes sobrevivientes de accidente cerebrovascular, ACV), incluyéndose $16.164 \mathrm{pa}-$ cientes $\geq 60$ años; con $2 / 3$ de éstos asignados a diuréticos y menos de $1 / 3$ asignados a ß-bloqueantes como monoterapias bien controladas. Sólo $33 \%$ de los asignados a diuréticos requirieron otra droga para controlar la hipertensión (HTA), pero aproximadamente $2 / 3$ de los asignados a $ß$-bloqueantes debieron asociar un diurético para lograrlo. El tratamiento diurético fue superior al de $ß$-bloqueantes con respecto a todos los resultados principales, analizados por intención de tratar*.

\section{Riesgo de eventos en pacientes tratados con diuréticos}

\begin{tabular}{lll}
\hline Eventos & OR & IC 95\% \\
\hline ACV & 0,61 & $0,51-0,72$ \\
\hline ACV Fatal & 0,67 & $0,49-0,90$ \\
\hline Enf. Coronaria & 0,74 & $0,64-0,85$ \\
\hline Mortalidad CV & 0,75 & $0,64-0,87$ \\
\hline Mortalidad Total & 0,86 & $0,77-0,96$ \\
\hline
\end{tabular}

En contraste los ß-bloqueantes solo redujeron los eventos cerebrovasculares, OR 0.75 (IC95\% 0.57-0.98) y no fueron efectivos para los demás resultados (0R próximos a 1). Los resultados fueron homogéneos entre los diferentes regímenes diuréticos (tiazidas solas o con ahorradores de potasio y clortalidona).

\section{Conclusiones}

A diferencia de los diuréticos, que siguen siendo el tratamiento de primera línea, los ß-bloqueantes, no deberían considerarse como terapia de primera línea de la HTA no complicada en los ancianos.

COMENTARIO

Numerosos estudios confirmaron la seguridad y eficacia de los diuréticos en la reducción de la morbimortalidad cardiovascular y total en hipertensos ancianos, pero los datos no eran tan claros con los ß-bloqueantes, por lo que el JNC ${ }^{1}$ dejó de recomendarlos en 1997 como terapia de primera línea en la HTA del anciano.

Este correcto meta-análisis prueba claramente la superioridad de los diuréticos sobre los $ß$-bloqueantes, éstos últimos no redujeron ni la enfermedad coronaria ni la mortalidad cardiovascular o total en estos pacientes. Además en el ensayo MRC ${ }^{2}$ los pacientes que recibían diuréticos asociados a $B$-bloqueantes tuvieron peores resultados que con diuréticos solos, demostrando que, aún normalizando la tensión arterial ( $T A$ ), los $B$-bloqueantes no mejoraban los resultados clínicos. En un reciente estudio caso-control ${ }^{3}$ el riesgo de muerte súbita cardíaca fue mayor en ancianos que recibían ß-bloqueantes (solos o con tiazidas) vs. otros antihipertensivos. Parte de la ineficacia de los ß-bloqueantes en este grupo etario puede explicarse por su débil efecto antihipertensivo (menos de $1 / 3$ de control como monoterapia). Además el abandono de ß-bloqueantes por efectos adversos en el estudio del MRC fue el doble que con diuréticos.

Los pacientes con diuréticos tuvieron menos eventos cardiovasculares que con ß-bloqueantes aún ajustando por el descenso de TA. Cabe destacar que la TA, que es un resultado intermedio*, no siempre se correlaciona con los resultados clínicos finales* de morbimortalidad. Esto podría deberse a una protección específica de los diuréticos o, lo

* Ver Glosario más preocupante, a que los ß-bloqueantes tengan un efecto cardiovascular desfavorable que contrarreste los beneficios del control de la TA en esta población. Un reciente subanálisis del estudio SHEP4 tampoco encontró efectos benéficos independientes atribuibles al atenolol o a la reserpina.

Ninguna evidencia sugiere que los $ß$-bloqueantes (solos o con diuréticos) reduzcan independientemente la morbimortalidad en ancianos; pero sí lo haría en pacientes jóvenes y de edad media en similar magnitud que con diuréticos ${ }^{5}$. Los ß-bloqueantes tienen menos efecto que otros antihipertensivos sobre una serie de fenómenos comunes en los ancianos como la HTA sistólica, la hipertrofia ventricular, la rigidez arterial o la hipertrofia vascular. Además pueden empeorar el filtrado glomerular, la tolerancia al ejercicio, el perfil lipídico, la obstrucción arterial y de la vía aérea. La principal hipótesis que podría explicar la ineficacia de los ßbloqueantes en la morbimortalidad de los ancianos es que la hemodinamia de éstos se caracteriza por un bajo gasto cardíaco y una elevada resistencia periférica; y la mayoría de los ß-bloqueantes actúan incrementando estos fenómenos, aún a largo plazo. Estos efectos hemodinámicos, opuestos a los deseables, podrían acelerar o empeorar las acciones de la HTA presumibles para esta edad.

En resumen, los ß-bloqueantes continúan siendo fundamentales en el post-infarto a cualquier edad, pero en los ancianos con HTA no complicada parecen exponerlos a algunos efectos adversos y a poco o ningún beneficio.

\section{Dr. Agustín Ciapponi}

Unidad de Medicina Familiar y Preventiva. Hospital Italiano de Buenos Aires.

\section{Referencias}

1. The Sixth Report of the Joint National Committee on Prevention, Detection, Evaluation, and Treatment of High Blood Pressure. Arch Intern Med. 1997;157:2413-2446.

2. MRC Working Party. Medical Research Council trial of hypertension in older adults; principal results. BMJ. 1992;304;405-412.

3. Hoes AW, Grobbee DE, Lubse J, et al. Diuretics, b-blockers and sudden cardiac death in hypertensive patients. Ann Intern Med 1995;123:481-487.

4. Kostis JB, Berge KG, Davis BR, et al. Effect of atenolol and reserpine on selected events in the the systolic hypertension in the elderly program (SHEEP). Am J of Hypertens. 1995;8(12 pt 1):1147-1153.

5. Staessen J, Fagard R, Amery A. Primary Prevention With Metoprolol in Patients With Hypertension [letter]. JAMA 1998;260:1713-1714. 


\section{El tamoxifeno es beneficioso en la mayoría de las pacientes con cáncer mamario temprano}

Tamoxifen for early breast cancer: an overview of the randomised trials. Early Breast Cancer Trialists Collaborative Group. Lancet 1998; 351: 1451-1466.

\section{Objetivo}

Revisar la evidencia científica en relación al beneficio del tamoxifeno en cuanto a reducción de recurrencias, de la mortalidad, y evaluar seguridad y duración del tratamiento en pacientes con cáncer de mama temprano.

\section{Fuente y selección de datos}

El grupo colaborativo contra el cáncer de mama procesó todos los datos publicados al respecto en estudios a leatorizados desde 1984 a 1996. Los trabajos analizados fueron divididos según la duración del tratamiento con tamoxifeno en tres categorías: 1 ; 2 ; y 5 años. Se identificaron 63 estudios, 55 de ellos cumplieron criterios de inclusión (involucrando 37.000 mujeres).

La información recolectada incluyó: edad, menopausia, compromiso ganglionar, medición de receptores hormonales, fechas de a leatorización, de tratamiento, aparición de cáncer contralateral o segundas neoplasias, recurrencia local o a distancia, muerte y sus causas.

\section{Resultados principales}

De las 37.000 mujeres, en las $\sim 8.000$ que tenían receptores estrogénicos negativos (RE-), el beneficio del tamoxifeno fue pequeño (RRR* $10 \%$, IC95\% 2-17\%, $\mathrm{p}<0.001$ ). En el resto de las mujeres (18.000 con $\mathrm{RE}+\mathrm{y} 12.000$ con RE no evaluados) los beneficios fueron estadística y clínicamente relevantes. Los siguientes datos se refieren a este grupo de 30.000 pacientes. En 10 años de seguimiento la reducción en las recurrencias fueron $21 \%, 29 \%$ y $47 \%$ en los grupos de tratamiento con tamoxifeno de 1, 2, y 5 años respectivamente y la reducción en la mortalidad global fue de $12 \%, 17 \%$ y $26 \%$ respectivamente. Tanto la recurrencia como la mortalidad disminuían a medida que se incrementaban los años de tratamiento ( $P<0.003$ para ambas).

La reducción relativa en la mortalidad fue similar tanto en mujeres con o sin compromiso ganglionar, pero las reducción absoluta de la mortalidad fue mayor en mujeres con compromiso axilar positivo. La reducción absoluta de recurrencias osciló entre un $4.7 \%\left(N N T^{*}=21\right)$ en estudios de un año y mujeres con ganglios negativos y un $15.2 \%$ (NNT=7) en estudios de cinco años y mujeres con ganglios positivos. La reducción absoluta de la mortalidad osciló entre un 3.4\% (NNT=30) en estudios de un año y mujeres sin ganglios y un $10.9 \%$ (NNT=9) en estudios de cinco años y mujeres con ganglios positivos. En todas las mujeres analizadas (incluyendo a las ER -) la reducción relativa en la aparición de cáncer de mama contralateral fue de $13 \%$, 26\% y $47 \%$ en los trabajos con 1, 2, 5 años de tamoxifeno respectivamente. La densidad de los receptores de estrógenos se relacionó directamente con mejoría en la sobrevida y las recurrencias. La posibilidad de una respuesta predecible al tamoxifeno no se relacionó con la presencia de receptores de progesterona, edad, menopausia, dosis, o si recibieron quimioterapia.

La incidencia de cáncer de endometrio fue aproximadamente el doble en los grupos con 102 años de tamoxifeno y el cuádruple en el grupo con 5 años de tratamiento. El exceso de riesgo absoluto promedio fue de 4 casos por 1000 pacientes a diez años $\left(N N D^{*}=250\right.$, es decir que se provocaría un cáncer de endometrio cada 250 mujeres tratadas a los diez años). La reducción absoluta en la incidencia de cáncer de mama contralateral fue casi el doble que el incremento absoluto en la incidencia de cáncer de endometrio. El tamoxifeno no aumentó las muertes por cáncer de colon u otras causas.

\section{Conclusiones}

El tamoxifeno como tratamiento adyuvante del cáncer de mama temprano mejora la sobrevida y disminuye las recurrencias en pacientes con receptores estrogénicos positivos o desconocidos. En pacientes con receptores negativos todavía es necesario seguir las investigaciones con estudios controlados.

\section{COMENTARIO}

Ya se ha demostrado que el uso de tamoxifeno adyuvante mejora la sobrevida a 10 años, sin embargo aún quedaban preguntas por responder: ¿Quiénes deben ser tratados?, ¿Por cuánto tiempo?, ¿Los riesgos del uso superan los beneficios en mujeres con bajo riesgo de recurrencia? El tamoxifeno reduce las recurrencias y la mortalidad en un $20-40 \%$ dependiendo de las características del tumor ${ }^{1}$. Las pacientes con tumores verdaderamente ER- no parecen beneficiarse con el tamoxifeno, pero si aquellas con ER+ (especialmente aquellos con alta intensidad).

Tanto las mujeres pre y postmenopausicas, como aquellas con axila positiva o no, se benefician con el tamoxifeno si poseen ER+. Esto nos hace pensar en cambiar algunos algoritmos de tratamientos que ya parecían estar fuertemente establecidos. La duración óptima del tratamiento con tamoxifeno parece ser de 5 años. Faltan estudios con adyuvancia hormonal más prolongada.

Una complicación poco frecuente es el cáncer de endometrio de bajo grado (en particular en post-menopáusicas), pero el riesgo por su aparición no llega a contrarrestar los beneficios en la sobrevida y de prevención de recurrencias.

Este importante, exhaustivo y meticuloso meta-análisis no demostró aumento de la incidencia de otras neoplasias o mortalidad por otras causas.

Una pregunta importante es si las mujeres pre-menopáusicas con ER+ deben ser tratadas con quimioterapia, tamoxifeno o ambos. Sólo un pequeño estudio demostró que el esquema Ciclofosfamida-Metrotexate-Fluouracilo fue superior al tamoxifeno. Otros trabajos no hallaron beneficios usando tamoxifeno luego de quimioterapia en pre-menopáusicas.

En post-menopáusicas, el tamoxifeno es superior a la quimioterapia, pero el interrogante es si la quimioterapia puede adicionar un beneficio adicional. Este meta-análisis sugiere que el agregado de quimioterapia provee un pequeño incremento de la reducción en recurrencias y mortalidad comparado con el tamoxifeno solamente. Resta evaluar si este beneficio marginal supera los efectos adversos, costos, y cómo esto afecta la calidad de vida de las mujeres con esta enfermedad. 